\title{
Blood pressure and age associated with silent autonomic dysreflexia during urodynamic examinations in patients with spinal cord injury
}

\author{
Y-H Huang ${ }^{1,2}$, L-I Bih ${ }^{1,2}$, J-M Liao ${ }^{3}$, S-L Chen ${ }^{1,4}$, L-W Chou ${ }^{5}$ and P-H Lin ${ }^{2}$
}

Study design: An observational study.

Objectives: To investigate the factors associated with silent autonomic dysreflexia (AD) during urodynamics (UD) in spinal cord injury (SCl) patients.

Setting: Taichung city, Taiwan, ROC.

Methods: Blood pressure (BP) and symptoms of AD were continuously monitored during UD, and systolic blood pressure (SBP) elevations $>20 \mathrm{mmHg}$ was considered an AD reaction. AD patients were divided into a symptomatic group and a silent group (without AD symptoms), and variables (basic demographic data, hemodynamic data and UD parameters) were compared.

Results: A total of 42 patients, 21 in symptomatic and 21 in silent AD groups, were analyzed. Symptomatic group had significantly greater diastolic blood pressure (DBP) increment and rates of SBP/DBP changes (change of BP divided by duration) as compared to the silent group (29.5 vs $21.7 \mathrm{mmHg} 4.8$ vs $2.3 \mathrm{mmHgmin}^{-1}$ and 2.8 vs $1.4 \mathrm{mmHgmin}^{-1}$ ) $(P<0.05)$. The cutoff points of these three variables, determined by receiver operating characteristic analysis, are: DBP increment of $21 \mathrm{mmHg}$ and rates of SBP/DBP change of 2.27 and $1.16 \mathrm{mmHg} \mathrm{min}^{-1}$. Age had a weak negative correlation with DBP change (Pearson's $r=-0.377, P=0.048$ ) and the silent group was significantly older than the symptomatic group (49.4 vs 40.1 years, $P<0.05$ ).

Conclusion: Patients with more symptomatic AD tended to have significant DBP elevation and more rapid SBP/DBP increments, and this was negatively correlated with age. Aging decreases AD symptoms and the magnitude of DBP elevation, possibly through the mechanism of decreased baroreceptor sensitivity. BP monitoring during UD and other invasive procedures is strongly recommended, especially for elderly $\mathrm{SCl}$ patients.

Spinal Cord (2013) 51, 401-405; doi:10.1038/sc.2012.155; published online 11 December 2012

Keywords: autonomic dysreflexia; baroreceptors; blood pressure; spinal cord injuries; urodynamics

\section{INTRODUCTION}

Patients with supra-sacral spinal cord injury (SCI) are reported to be susceptible to autonomic dysreflexia (AD), especially those injured at the T6 level or higher, and have a prevalence of nearly half to threequarters. $^{1,2} \mathrm{AD}$ is not an all-or-none reaction. It varies in intensity from asymptomatic to a life-threatening emergency. ${ }^{1,3}$ Serious morbidity, like consciousness change, visual disturbances, seizures, intracranial hemorrhage or even death, is primarily associated with sudden and marked rises in blood pressure (BP). ${ }^{4-6}$ Symptoms (that is, headache, facial flushing, chills or sweating) are usually used as warning signs of BP elevation, although sometimes there is no presenting symptoms when $\mathrm{BP}$ is elevated, a condition referred to as silent AD. ${ }^{6}$ In such cases, patients and physicians may miss the ideal time to manage the underlying strong afferent stimuli until serious sequelae ensue.

As stimuli from the lower urinary tract are the most common causes of $\mathrm{AD}$, reportedly in $75-85 \%$ of cases, ${ }^{2,3}$ the routine urodynamic (UD) study of these patients offers a controlled environment to observe the $\mathrm{AD}$ reactions. Several previous researches have investigated $\mathrm{BP}$ responses in UD examinations to further understand $\mathrm{AD}$ reactions and the associated factors. ${ }^{3,6-9}$ Some of these have also found that the proportion of patients presenting with silent $\mathrm{AD}$ ranges from 35 to $43 \%{ }^{6,8,9}$ However, only one, the study by Linsenmeyer et al., ${ }^{6}$ investigated the differential variables between symptomatic and silent AD. They found that no factor was significant, including BP values, levels of neurologic injury, length of injury, voiding pressures and bladder capacity. However, that study used a fixed level of BP (systolic BP $160 \mathrm{mmHg}$ and diastolic BP $90 \mathrm{mmHg}$ ) as a definition of $\mathrm{AD}$, which is different from more recent concepts that define increments of $20-40 \mathrm{mmHg}$ from resting SBP as an $\mathrm{AD}$ episode. ${ }^{10-12}$

As silent $\mathrm{AD}$ may be an important clinical concern regarding the health of the SCI patients, the present study sought to discover the factors associated with $\mathrm{AD}$ symptoms and investigate the mechanisms

${ }^{1}$ School of Medicine, Chung Shan Medical University, Taichung, Taiwan; ${ }^{2}$ Department of Physical Medicine and Rehabilitation, Chung Shan Medical University Hospital, Taichung, Taiwan; ${ }^{3}$ Department of Physiology, Chung Shan Medical University, Taichung, Taiwan; ${ }^{4}$ Department of Urology, Chung Shan Medical University Hospital, Taichung, Taiwan and ${ }^{5}$ Department of Physical Medicine and Rehabilitation, China Medical University Hospital, Taichung, Taiwan 
underlying silent $\mathrm{AD}$. In this study, $\mathrm{BP}$ and its symptoms were monitored throughout each UD examination, and an SBP increment $>20 \mathrm{mmHg}$ was defined as an $\mathrm{AD}$ reaction.

\section{MATERIALS AND METHODS}

From September 2010 to March 2012, 132 adult supra-sacral SCI patients who received UD examinations from Chung Shan Medical University Hospital were screened. The inclusion criteria were 18-65 years of age, neurologic injury level at T6 or above with stable condition, and not in the spinal shock stage (judged by increased muscle tone and presence of detrusor contraction or sphincter spasticity on UD). The exclusion criteria included neurologic diseases other than SCI, previous genito-urinary disease or surgery, current urinary tract infection, history of cardiovascular disease (including hypertension and peripheral vascular disease) and multiple injury levels. After screening, 101 patients were included for further investigation.

The classification of neurologic injury types (level and completeness) were according to the American Spinal Injury Association criteria. ${ }^{13}$ An SBP elevation $>20 \mathrm{mmHg}$ was defined as $\mathrm{AD}$ reaction, and these patients were enrolled for the final analysis. ${ }^{12}$

The execution and reading of UD examinations followed the standards of the International Continence Society. ${ }^{14}$ Cystourethrometry was performed by a triple-lumen catheter with continuous filling of isotonic saline at a rate of $30 \mathrm{mlmin}{ }^{-1}$. Electromyography of the external urethral sphincter was obtained via concentric needle electrodes. Filling was stopped if one of the following conditions presented: (1) patient reported a sensation of fullness, (2) spontaneous urine leakage, (3) infused volume reached $500 \mathrm{ml}$, (4) BP reached a dangerous level (SBP $180 \mathrm{mmHg}$ or DBP $110 \mathrm{mmHg}$ ) and (5) intolerable AD symptoms.

$\mathrm{BP}$, pulse rate and any $\mathrm{AD}$ symptoms (that is, headache, facial flushing, chills or sweating) were recorded before UD examination, every 2 minutes during bladder filling/voiding and after the examination. The BP and pulse rate measurements were obtained from an automatic sphygmomanometer with an inflatable cuff wrapped around one of the patient's upper arms. Each measurement took about 30-40 s. Maximal SBP and DBP changes were calculated as maximal values subtracted by the baseline value. Maximal changes of SBP/DBP divided by duration of time was defined as the rates of SBP/DBP change.

Other variables suspected of having an association with the presentation of $\mathrm{AD}$ symptoms were also compared between the symptomatic and silent $\mathrm{AD}$ groups. These include gender, age, injury duration, injury level, completeness of injury, maximal intra-vesical pressure, maximal urethral pressure, onset volume and duration of detrusor contraction (DC), bladder compliance, and types of detrusor sphincter dyssynergia (DSD). Bladder compliance was calculated from the period between the initiation of filling and before the start of any DC or the end of filling, if there was no DC. The DSD was classified into intermittent and continuous types according to the consistency of sphincter contraction. ${ }^{15}$ Duration of DC was the interval between the onset of DC and urine leakage or termination of examination.

In the patients with elevated BP during UD examination, their BP usually increased in a continuous and progressive way until the bladder was evacuated. In order to better understand the stimuli most strongly associated with $\mathrm{AD}$ reaction during examination, SBP difference before and after each UD event (that is, onset of DC, onset of DSD, maximal urethral pressure, maximal intravesical pressure, urine leakage, and maximal capacity) were compared. It is calculated as the first SBP record after an UD event subtracted by the last record before this event.

Differences in distribution of categorical variables between symptomatic and silent $\mathrm{AD}$ were analyzed by $\chi^{2}$-test, whereas differences in continuous variables between these two groups were analyzed by $t$-test. If any continuous variable showed significant difference between these two groups, we used receiver operating characteristic curve analysis to calculate the best cutoff point (that is, maximal value of sensitivity plus specificity) between them. Differences in BP changes of UD events were analyzed by repeat measure analysis of variance (ANOVA). The level of significance was set at $P<0.05$.

\section{Statement of ethics}

The hospital's Ethics Committee approved the study and all patients provided prior written informed consent.

\section{RESULTS}

A total of 42 patients were selected by the aforementioned criteria and those with SBP increments $>20 \mathrm{mmHg}$ entered the final statistical analysis. The prevalence of AD in our SCI patients with injury level at T6 or above was $41.7 \%(42 / 101)$. Among these 42 patients, 21 (50\%) complained of one or more $\mathrm{AD}$ symptoms (symptomatic $\mathrm{AD}$ group) and the remaining $21(50 \%)$ had no complaints when BP elevated (silent $\mathrm{AD}$ group). The presenting symptoms were sweating in 10 , headache in 9, facial flushing in 2 and chills in 2. The basic data of these 42 patients and the comparisons of these variables between the symptomatic and silent $\mathrm{AD}$ groups were shown in Table 1 . The symptomatic group was significantly younger than the silent group (40.1 \pm 11.7 vs $49.1 \pm 10.4$ years). There was no difference in other basic variables. The other 59 patients without BP elevation $>20 \mathrm{mmHg}$ during UD had no complaints of AD symptoms.

Comparisons of hemodynamic and UD parameters between the symptomatic and silent $\mathrm{AD}$ group were shown in Tables 2 and 3 . Symptomatic AD patients showed more significant DBP increments $(29.5 \pm 9.4$ vs $21.7 \pm 7.9 \mathrm{mmHg})$ and greater rate of SBP/DBP elevation than the silent $\mathrm{AD}$ patients (rates of SBP change $4.8 \pm 2.8 v \mathrm{~s}$ $2.3 \pm 1.3 \mathrm{mmHg} \mathrm{min}^{-1}$; rates of DBP change $2.8 \pm 1.1$ vs $\left.1.4 \pm 0.9 \mathrm{mmHg} \mathrm{min}^{-1}\right)(P<0.05)$. Receiver operating characteristiccurve analysis showed that the best cutoff points to predict patients having $\mathrm{AD}$ symptoms are: $\mathrm{DBP}$ increment of $21 \mathrm{mmHg}$ (sensitivity 86 ; specificity $69 \%$ ), rate of SBP change of $2.27 \mathrm{mmHg} \mathrm{min}^{-1}$ (sensitivity 79; specificity $80 \%$ ) and rate of DBP change of $1.16 \mathrm{mmHg} \mathrm{min}^{-1}$ (sensitivity 100; specificity 69\%). (Figure 1) The symptomatic group had non-significant increases in maximal SBP/ DBP value, SBP increment, maximal urethral pressure, bladder stiffness and chance of continuous DSD. A sage was a factor associated

Table 1 Comparison of basic demographic data between symptomatic and silent AD groups

\begin{tabular}{lccc}
\hline & Total & Symptomatic AD & Silent $A D$ \\
\hline Number & 42 & 21 & 21 \\
Age & $44.8 \pm 12.9$ & $40.1 \pm 11.7$ & $49.4 \pm 11.7$ \\
Mean \pm s.d. (range) & $(22-65)$ & $(22-63)^{*}$ & $(27-65)^{*}$ \\
Gender & & & \\
$\quad$ Male & 35 & 18 & 17 \\
Female & 7 & 3 & 4 \\
Injury duration & $18.3 \pm 38.1$ & $15.8 \pm 18$ & $20.6 \pm 45.9$ \\
(months) & $(1.5-180)$ & $(2-60)$ & $(1.5-180)$
\end{tabular}

Mean \pm s.d. (range)

Injury level

$\begin{array}{lccc}\text { Cervical } & 35 & 18 & 17 \\ & 7 & 3 & 4\end{array}$

Completeness of injury

$\begin{array}{llll}\text { Complete } & 18 & 10 & 8\end{array}$

Incomplete

24

11

13

Abbreviations: $A D$, autonomic dysreflexia; s.d., standard deviation. ${ }^{*} P<0.05$. 
with symptomatic presentation, the correlations of age and other hemodynamic factors (that is, baseline and maximal BP, maximal changes of BP and rates of BP elevation) were further analyzed. The

Table 2 Comparison of hemodynamic parameters between symptomatic and silent groups

\begin{tabular}{|c|c|c|c|c|}
\hline & Total & Symptomatic $A D$ & Silent $A D$ & $\mathrm{P}$ value \\
\hline \multicolumn{5}{|c|}{ Baseline SBP ( $m m H g)$} \\
\hline Mean \pm s.d. & $116.2 \pm 14.0$ & $115.5 \pm 16.5$ & $116.9 \pm 10.3$ & 0.798 \\
\hline Range & $70 \sim 140$ & $70 \sim 139$ & $81 \sim 140$ & \\
\hline \multicolumn{5}{|c|}{ Baseline DBP ( $\mathrm{mmHg})$} \\
\hline Mean \pm s.d. & $76.4 \pm 9.9$ & $76.5 \pm 14.4$ & $76.3 \pm 7.5$ & 0.755 \\
\hline Range & $42 \sim 92$ & $42 \sim 92$ & $51 \sim 85$ & \\
\hline \multicolumn{5}{|c|}{ Maximal SBP (mmHg) } \\
\hline Mean \pm s.d. & $163.6 \pm 24.7$ & $168.4 \pm 32.7$ & $159.1 \pm 13.9$ & 0.266 \\
\hline Range & $105 \sim 208$ & $105 \sim 208$ & $121 \sim 179$ & \\
\hline \multicolumn{5}{|c|}{ Maximal DBP (mmHg) } \\
\hline Mean \pm s.d. & $101.4 \pm 14.1$ & $104.7 \pm 16.3$ & $98.1 \pm 10.1$ & 0.235 \\
\hline Range & $72 \sim 123$ & $72 \sim 123$ & $79 \sim 115$ & \\
\hline \multicolumn{5}{|c|}{ Maximal SBP change $(\mathrm{mmHg})$} \\
\hline Mean \pm s.d. & $45.4 \pm 18.1$ & $51.6 \pm 24.3$ & $40.6 \pm 13.6$ & 0.159 \\
\hline Range & $22 \sim 94$ & $22 \sim 94$ & $23 \sim 66$ & \\
\hline \multicolumn{5}{|c|}{ Maximal DBP change $(\mathrm{mmHg})$} \\
\hline Mean \pm s.d. & $24.9 \pm 9.7$ & $29.5 \pm 9.4^{*}$ & $21.7 \pm 7.9^{*}$ & $0.042^{*}$ \\
\hline Range & $11 \sim 46$ & $11 \sim 46$ & $12 \sim 35$ & \\
\hline \multicolumn{5}{|c|}{ Maximal PR change (beats min $^{-1}$ ) } \\
\hline Mean \pm s.d. & $-5.7 \pm 11.9$ & $-6.8 \pm 14.7$ & $-4.8 \pm 8.5$ & 0.641 \\
\hline Range & $-20 \sim 32$ & $-20 \sim 32$ & $-19 \sim 18$ & \\
\hline \multicolumn{5}{|c|}{ Rate of SBP change $\left(\mathrm{mmHg} \mathrm{min}^{-1}\right)$} \\
\hline Mean \pm s.d. & $3.5 \pm 2.6$ & $4.8 \pm 2.8^{*}$ & $2.3 \pm 1.3^{*}$ & $0.004^{*}$ \\
\hline Range & $1.25 \sim 11.5$ & $1.55 \sim 11.5$ & $1.25 \sim 6.60$ & \\
\hline \multicolumn{5}{|c|}{ Rate of $D B P$ change $\left(\mathrm{mmHg} \mathrm{min}^{-1}\right)$} \\
\hline Mean \pm s.d. & $2.1 \pm 1.2$ & $2.8 \pm 1.1^{*}$ & $1.4 \pm 0.9 *$ & 0.001 * \\
\hline Range & $0.31 \sim 5.33$ & $1.31 \sim 5.33$ & $0.31 \sim 3.50$ & \\
\hline
\end{tabular}

Abbreviations: AD, autonomic dysreflexia; DBP, diastolic blood pressure; SBP, systolic blood pressure; PR, pulse rate; s.d., standard deviation.

$* P<0.05$. only correlation found between age and hemodynamic factors was a weak one with DBP change. (Pearson's $r=-0.377, P=0.048$ ).

The results of comparing SBP changes during different UD events were shown in Table 4. We found SBP increase was most prominent at the onset of DC and DSD. The AD symptoms did not always present simultaneously during the periods of significant BP elevation and half appeared after BP elevation.

\section{DISCUSSION}

In this study, the prevalence of $\mathrm{AD}$ is $41.7 \%$ in our SCI patients with injury level at T6 or above. This is similar to a previous report using the same definition of $\mathrm{AD}$ reaction $(\mathrm{SBP}$ increment $>20 \mathrm{mmHg}) .^{12}$ Among these patients with signs of $\mathrm{AD}, 50 \%$ were symptomatic and $50 \%$ were silent. This percentage of silent $\mathrm{AD}$ is higher than three previous studies that reported 35,38 and $43 \%$ respectively. ${ }^{6,8,9}$ This discrepancy may be due to the different criteria of $\mathrm{AD}$ in these studies. Two of them used definite levels of SBP/DBP values as $160 / 100$ and $150 / 100 \mathrm{mmHg}$, respectively, whereas one used an SBP increment percentage of $30 \%$ of baseline value. As tetraplegic SCI

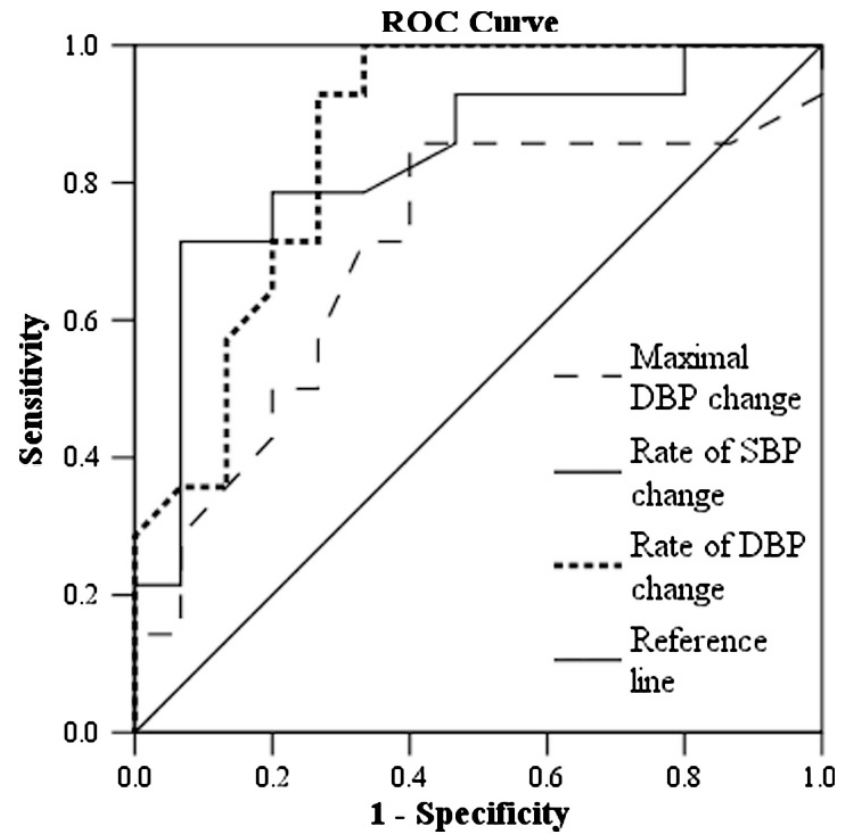

Figure 1 Receiver operating characteristic curve analysis of maximal DBP change, rate of SBP change, and rate of DBP change.

Table 3 Comparison of UD parameters between symptomatic and silent groups

\begin{tabular}{|c|c|c|c|c|}
\hline & Total & Symptomatic $A D$ & Silent $A D$ & $\mathrm{P}$ value \\
\hline Volume of DC onset (ml) & $193.9 \pm 111.5$ & $204.1 \pm 106.0$ & $184.1 \pm 103.8$ & 0.768 \\
\hline Duration of DC (min) & $7.0 \pm 4.1$ & $6.4 \pm 3.4$ & $7.4 \pm 4.5$ & 0.481 \\
\hline Maximal intravesical pressure $(\mathrm{cmH} 2 \mathrm{O})$ & $76.3 \pm 33.6$ & $78.6 \pm 31.0$ & $74.1 \pm 36.1$ & 0.659 \\
\hline Maximal urethral pressure $(\mathrm{cmH} 2 \mathrm{O})$ & $85.6 \pm 37.0$ & $86.9 \pm 35.6$ & $75.1 \pm 33.7$ & 0.081 \\
\hline Compliance $\left(\mathrm{ml} \mathrm{cm}^{-1} \mathrm{H} 2 \mathrm{O}\right)$ & $25.1 \pm 21.7$ & $22.9 \pm 15.2$ & $29.8 \pm 24.2$ & 0.145 \\
\hline Type of DSD (no.) & & & & 0.054 \\
\hline No DSD & 1 & 0 & 1 & \\
\hline Intermittent DSD & 10 & 2 & 8 & \\
\hline Continuous DSD & 31 & 19 & 12 & \\
\hline
\end{tabular}

Abbreviations: AD, autonomic dysreflexia; DC, detrusor contraction; DSD, detrusor sphincter dyssynergia; UD, urodynamic. 
Table 4 Comparisons of SBP changes during UD events

\begin{tabular}{|c|c|c|c|c|c|c|}
\hline & Onset of $D C$ & Onset of $D S D$ & Max Pure & Max Pves & Urine leakage & Maximal capacity \\
\hline SBP changes (mmHg) & $13.7 \pm 12.2^{*}$ & $13.1 \pm 13.5^{*}$ & $4.6 \pm 13.8$ & $5.0 \pm 12.8$ & $-1.2 \pm 14.5$ & $-3.1 \pm 12.0$ \\
\hline Mean \pm s.d. & & & & & & \\
\hline
\end{tabular}

patients usually have lower BP level than able-bodied persons, often at the range of $90 / 60 \mathrm{mmHg}$, the criteria of $\mathrm{AD}$ in these three studies are stricter than that of the current study and thus, might result in a higher rate of symptomatic $\mathrm{AD}$ and lower rate of silent $\mathrm{AD}$. Moreover, two of these studies mentioned that some patients in the group that they defined as normotensive showed AD symptoms. ${ }^{6,9}$ No one in the non-AD group in the present study had any $\mathrm{AD}$ symptoms.

Both this study and the previous report by Linsenmeyer et al. ${ }^{6}$ reveal that the absolute levels of BP is not crucial to symptomatic presentation. Although the symptomatic $\mathrm{AD}$ group here has slightly higher values of maximal SBP and $\mathrm{DBP}$ than the silent $\mathrm{AD}$ group, the difference is not significant. That is to say, silent $\mathrm{AD}$ patients may have dangerously high levels of SBP, reaching $180 \mathrm{mmHg}$ in this study (Table 2). On the other hand, we found the magnitude of BP increments, especially DBP, and the rates of $\mathrm{BP}$ changes are factors associated with the presentation of $\mathrm{AD}$ symptoms. After further receiver operating characteristiccurve analysis, patients with DBP increment reaching $21 \mathrm{mmHg}$, rate of SBP change reaching $2.27 \mathrm{mmHg} \mathrm{min}^{-1}$ and rate of DBP change reaching $1.16 \mathrm{mmHg} \mathrm{min}^{-1}$, were found to have greater chance to be symptomatic. On the contrary, patients with BP changes lower than these values tended to be silent. We think this phenomenon could be partially explained by the physiology of baroreceptor reflex.

$\mathrm{AD}$ is a phenomenon initiated by a sudden rise in sympathetic tone with BP elevation induced by strong afferent stimuli below the injury level. While BP is elevated, some patients may experience uncomfortable symptoms, including headache, sweating and flushing, which may be associated with passively increased blood flow or vasodilatation above the lesion levels. ${ }^{1,2,16}$ In this chain of reaction, baroreceptors mediated reflex arcs might have some important roles. ${ }^{1,2,16}$ Baroreceptors are mechano-sensitive terminals triggered by both the magnitude and rate of change in arterial pressure level, which is usually referred to as the mean arterial pressure, a composition of $1 / 3 \mathrm{SBP}$ and $2 / 3 \mathrm{DBP} .{ }^{17}$ This may explain in part why magnitude of DBP increment, as well as the rate of BP changes, have a more important role than the magnitude of SBP changes in inducing symptoms.

In the present study, many factors, including injury patterns (level, duration and completeness) and UD parameters (onset/ duration of DC, maximal intra-vesical/urethral pressure, compliance and type of DSD), are found to be not associated with AD symptoms. This finding is similar to that in the study of Linsenmeyer et al. ${ }^{6}$, wherein the variables included were level and length of injury, bladder capacity and voiding pressure. However, the current study notes that patients without symptoms are significantly older than those with symptoms, and this may result partly from the weak negative correlation of age with DBP change (Pearson's $r=-0.377$ ). Thus, older patients have less DBP increase during UD and lower chances of being symptomatic. The effect of age on BP has been reported on in previous literature reports, noting that despite the continuous increase in SBP with age, DBP reaches its maximum at the age of 55 years and then begins to fall. ${ }^{18}$ Stiffening of the aorta with diminished elastic recoil during aging may cause the runoff of blood during systole, resulting in reduced blood volume within the aorta and lower diastolic pressure. ${ }^{19}$ The other possible cause of aging effect on symptomatic presentation is the reduction of baroreceptor sensitivity when patients get older. ${ }^{20}$ These results serve as a reminder that older SCI patients have higher risk of silent $\mathrm{AD}$ and should be closely monitored as regards their BP during invasive procedures or even during voiding and defecation.

During UD examination, a previous research study reported that BP increase begins mostly at the peak of uninhibited DC or at maximal bladder capacity. ${ }^{9}$ As elevation of BP during UD examination is a gradual but continuous pattern, it is difficult to determine the beginning or onset of BP increment. By comparing SBP difference before and after each UD event, this study reveals that the most prominent changes in SBP elevation occur at the onsets of DC and DSD. This phenomenon may be supported by the theory of Perkash et al. ${ }^{7}$ in 1979 . They proposed that $\mathrm{AD}$ is a spinal reflex mediated by afferents arising from the bladder wall near the bladder neck. These structures are noncompliant in patients with abnormal holding reflex-sphincter dyssynergia and convey excessive stimulus impulses when being stretched. On the other hand, as AD is a chain of reaction, symptoms present simultaneously with or later than $\mathrm{BP}$ elevation, as shown in the current study.

This study has some limitations that are worth noting. First, the total number of included subjects was only 42 and some variables seemed different but not significant. Second, a fixed infusion rate of $30 \mathrm{ml} \mathrm{min}^{-1}$ was used. As such, it could not be seen if the infusion rate was an influencing factor of symptomatic presentation. As $\mathrm{AD}$ is thought to be induced by stretching of the bladder wall or neck, the infusion rate may have some influence on $\mathrm{AD}$ symptoms. Lastly, $\mathrm{AD}$ symptoms were recorded according to subjective complaints. More objective measurements like sympathetic skin response or heart-rate variability could be used in the future.

\section{CONCLUSIONS}

Under the definition of SBP elevation $>20 \mathrm{mmHg}$, SCI patients, injured at or above $\mathrm{T} 6$ level, with $\mathrm{AD}$ reactions have a $50 \%$ rate of silent $\mathrm{AD}$. Elevation of $\mathrm{BP}$ is mostly significant at the onsets of $\mathrm{DC}$ and DSD during UD examination. Patients with symptomatic AD were associated with more significant DBP elevation and more rapid SBP/DBP increase. Aging patients had greater chance to have silent $\mathrm{AD}$ and this might be explained with decreased DBP elevation and possibly blunting of baroreceptors. BP monitoring during UD and other invasive procedures or even during voiding, is strongly recommended especially for aged SCI patients.

\section{DATA ARCHIVING}

There were no data to deposit.

\section{CONFLICT OF INTEREST}

The authors declare no conflict of interest. 


\section{ACKNOWLEDGEMENTS}

This study is supported by a research grant from the Chung Shan Medical University Hospital (grant no. CSH 2009-B-005).

1 Karlsson AK. Autonomic dysreflexia. Spinal Cord 1999; 37: 383-391.

2 Blackmer J. Rehabilitation medicine: 1. Autonomic dysreflexia. CMAJ 2003; 169: 931-935.

3 Lindan $\mathrm{R}$, Joiner $\mathrm{E}$, Freehafer AA, Hazel $\mathrm{C}$. Incidence and clinical features of autonomic dysreflexia in patients with spinal cord injury. Paraplegia 1980; 18: 285-292.

4 Yarkony GM, Katz RT, Wu YC. Seizures secondary to autonomic dysreflexia. Arch Phys Med Rehabil 1986; 67: 834-835.

5 Eltorai I, Kim R, Vulpe M, Kasravi H, Ho W. Fatal cerebral hemorrhage due to autonomic dysreflexia in a tetraplegic patient: case report and review. Paraplegia 1992; 30: 355-360.

6 Linsenmeyer TA, Campagnolo DI, Chou IH. Silent autonomic dysreflexia during voiding in men with spinal cord injuries. J Urol 1996; 155: 519-522.

7 Perkash I. Pressor response during cystomanometry in spinal injury patients complicated with detrusor-sphincter dyssynergia. J Urol 1979; 121: 778-782.

8 Curt A, Nitsche B, Rodic B, Schurch B, Dietz V. Assessment of autonomic dysreflexia in patients with spinal cord injury. J Neurol Neurosurg Psychiatry 1997; 62: 473-477.

9 Giannantoni A, Di Stasi SM, Scivoletto G, Mollo A, Silecchia A, Fuoco U et al. Autonomic dysreflexia during urodynamics. Spinal Cord 1998; 36: 756-760.

10 Consortium for Spinal Cord Medicine. Acute management of autonomic dysreflexia: individuals with spinal cord injury presenting to health-care facilities. J Spinal Cord Med 2002; 25(Suppl 1): S67-S88.
11 Krassioukov A, Warburton DE, Teasell R, Eng JJ. A systematic review of the management of autonomic dysreflexia after spinal cord injury. Arch Phys Med Rehabil 2009; 90: 682-695.

12 Huang YH, Bih LI, Chen GD, Lin CC, Chen SL, Chen WW. Autonomic dysreflexia during urodynamic examinations in patients with suprasacral spinal cord injury. Arch Phys Med Rehabil 2011; 92: 1450-1454.

13 American Spinal Injury Association and International Medical Society of Paraplegia e. Reference Manual of the International Standards for Neurological Classification of Spinal Cord Injury. American Spinal Injury Association: Chicago, IL, 2003.

14 Haylen BT, de Ridder D, Freeman RM, Swift SE, Berghmans B, Lee J et al. An International Urogynecological Association (IUGA)/International Continence Society (ICS) joint report on the terminology for female pelvic floor dysfunction. Neurourol Urodyn 2010; 29: 4-20.

15 Weld KJ, Graney MJ, Dmochowski RR. Clinical significance of detrusor sphincter dyssynergia type in patients with post-traumatic spinal cord injury. Urology 2000; 56: 565-568.

16 Barton CH, Khonsari F, Vaziri ND, Byrne C, Gordon S, Friis R. The effect of modified transurethral sphincterotomy on autonomic dysreflexia. J Urol 1986; 135: $83-85$.

17 Dampney RAL. Cardiovascular and respiratory reflexes: physiology and pharmacology. In: Low PA and Benarroch EE (eds). Clinical Autonomic Disorder, 3rd edn. Lippincott Williams \& Wilkins: Philadelphia, 2008.

18 Franklin SS, Wt Gustin, Wong ND, Larson MG, Weber MA, Kannel WB et al. Hemodynamic patterns of age-related changes in blood pressure. The Framingham Heart Study. Circulation 1997; 96: 308-315

19 Folkow B. Structure and function of the arteries in hypertension. Am Heart J 1987; 114(4 Pt 2): 938-948.

20 Lipsitz LA, Novak V. Aging and Autonomic Function. In: Low PA and Benarroch EE (eds). Clinical Autonomic Disorders, 3rd edn. Lippincott Williams \& Wilkins: Philadelphia, 2008. 